\title{
Is lipid peroxidation relevant to atherogenesis?
}

\author{
Jay W. Heinecke
}

Commentary

See related article

this issue, pages 213-220.

Departments of Medicine and Molecular Biology and Pharmacology, Washington University School of Medicine, Box 8046, 660 South Euclid Avenue, St. Louis, Missouri 63110, USA. Fax: (314) 362-0811; E-mail: heinecke@im.wustl.edu.

Many lines of evidence suggest that LDL, the major carrier of blood cholesterol, becomes atherogenic as a result of oxidation (reviewed in refs. 1-3). One of the most persuasive observations is that structurally unrelated lipid-soluble antioxidants inhibit atherogenesis in animal models of hypercholesterolemia. For example, probucol, butylated hydroxytoluene, and $N, N^{\prime}$-diphenyl-phenylenediamine all inhibit LDL oxidation in vitro, and retard the progression of atherosclerosis in hypercholesterolemic rabbits, mice, and quail. Moreover, probucol exerts a significant effect on thoracic atherosclerosis in primates with dietinduced hypercholesterolemia. These observations may be relevant to human disease, because a recent clinical trial of vitamin $\mathrm{E}$ suggests that antioxidant therapy inhibits the clinical sequelae of atherosclerosis (4).

Early in vitro experiments revealed that endothelial cells, smooth muscle cells, monocytes, and macrophages the major cellular components of atherosclerotic lesions - all render LDL atherogenic (5-7). LDL modification required iron or copper in the medium, generated dicarbonyl compounds that could react with thiobarbituric acid, and was inhibited by lipidsoluble antioxidants. This led to the hypothesis that lipid peroxidation plays a key role in rendering LDL atherogenic. This proposal was supported by chemical studies of oxidized LDL (8) and by the immunohistochemical detection of protein-bound adducts of malondialdehyde and other end-stage lipid oxidation products in atherosclerotic lesions $(9,10)$.

The next key observation was the demonstration that probucol, a lipidsoluble compound carried in LDL, dramatically reduced atherosclerosis in most hypercholesterolemic animals (11-13). Also, LDL isolated from animals and humans treated with probucol exhibited marked resistance to LDL oxidation ex vivo (14). Similar findings were reported for other lipid-soluble antioxidants (reviewed in refs. 3, 15). This has led to an explosion of interest in measuring the resistance of lipoproteins isolated from animals and humans subjected to various therapeutic interventions. The underlying assumption is that LDL's capacity to resist lipid peroxidation ex vivo correlates with the effectiveness of antioxidant intervention and the inhibition of vascular disease.

This appealing hypothesis is attracting skepticism because of recent experimental results. A probucol analogue, bis(3,5-di-tert-butyl-4-hydroxyphenylether)propane, failed to prevent atherosclerosis in rabbits, even though it strongly inhibited LDL oxidation in vitro (16). Because the LDL from the analogue-treated animals was less easily oxidized than that from untreated animals, it was suggested that LDL must reach a threshold of resistance to oxidation before atherogenesis is interrupted. However, several other studies have shown that LDL's resistance to oxidation ex vivo fails to predict the ability of antioxidant therapy to inhibit atherosclerosis, suggesting that this measure of antioxidant activity is of limited biological relevance $(3,15)$.

In this issue of the JCI, Witting et al. raise further provocative questions regarding the role of lipid peroxidation in LDL oxidation and atherogenesis. They demonstrate that the probucol metabolite bisphenol appears to completely block lipid peroxidation in the artery wall of hypercholesterolemic rabbits, without affecting the extent of atherosclerosis (17). The degree of lipid peroxidation in the atherosclerotic tissue also failed to correlate with the extent of lesion formation. The authors monitored lipid peroxidation with a sensitive and specific analytical technique, using HPLC to quantify the production of hydroxides and hydroperoxides of cholesteryl esters and triglycerides.

Before we overturn the dogma of lipid peroxidation, it is important to note several limitations of the current study.
First, lipid peroxidation was assessed at a single time point relatively late in the disease process. Therefore, failure to detect lipid peroxidation products at this late stage in probucol- or bisphenoltreated animals does not exclude the possibility that such products were present and active early in the disease process. Second, it is possible that levels of lipid peroxidation products below those detectable in the HPLC assay promoted atherosclerosis. Third, cholesterol levels were approximately 30\% higher in animals treated with bisphenol rather than probucol. This elevation in plasma cholesterol, a known atherogenic agent, may have masked a small but significant effect of the bisphenol in retarding lesion formation. Fourth, it is always technically difficult to ensure that lipid peroxidation products in tissues are extracted and detected quantitatively, even though this issue was carefully addressed in the study. Finally, it will be important to extend these findings to other animal models of atherosclerosis. One complicating factor in many explorations of the oxidation hypothesis is that a given antioxidant can have varying effects in different animal models $(3,11-13,15,16)$.

What are the implications of these results for the LDL oxidation hypothesis? It is important to note that antioxidants generally have been fed to animals in large amounts, typically $1 \%$ of the diet; therefore they reach extremely high concentrations in plasma and tissue. Moreover, recent studies have shown that lower levels of dietary probucol fail to inhibit atherosclerosis in hypercholesterolemic rabbits despite significantly reducing LDL oxidation ex vivo (18). Collectively, these observations suggest that nonspecific lipid-soluble antioxidants may not be the ideal therapeutic agents for preventing LDL oxidation and atherosclerosis.

Another crucial question is the identity of the oxidation products that promote atherosclerosis. If peroxidized fatty acids are not atherogenic, what is? Other oxidized lipids, including $\mathrm{F}_{2}$-iso- 
prostanes, cholesterol 7- $\beta$-hydroperoxide, and phospholipids with oxidatively cleaved side chains, have been detected in atherosclerotic lesions (19-21). Moreover, vitamin $\mathrm{E}$ therapy lowers plasma and aortic wall levels of $\mathrm{F}_{2}$-isoprostanes and inhibits atherosclerosis in hypercholesterolemic mice (19), suggesting that lipid oxidation products may contribute to vascular disease. Thus, the lipid hydroxides and hydroperoxides measured by Witting et al. may not be the relevant oxidation products. It also is possible that oxidation of proteins, proteoglycans, or nucleic acids plays a role in atherosclerosis. Certain protein oxidation products are present at markedly higher levels in human atherosclerotic lesions than in normal aortic tissue (reviewed in ref. 22), and the ability of antioxidants to inhibit protein oxidation may differ markedly from their ability to inhibit lipid oxidation. These findings emphasize the importance of analytical techniques, such as HPLC and mass spectrometry, that can quantify tissue levels of oxidation products. Using quantitative methods to detect specific molecular markers in atherosclerotic plaque should enable us to better assess the ability of antioxidants to block atherosclerosis and oxidation in the artery wall.

Finally, to understand the effects of antioxidants on atherosclerosis, it will be important to define the biochemical pathways that promote oxidative reactions in vivo (22). The most widely studied mechanism for LDL oxidation in vitro involves free, redox-active metal ions (5-7), but recent results have cast doubt on the physiological relevance of such reactions (23). Therefore, using in vitro findings to speculate about the effectiveness of antioxidants in vivo may be counterproductive. Findings from mouse models with targeted inactivation of specific genes may be a better guide. This approach has provided com- pelling evidence that lipoxygenase plays a role in promoting atherosclerosis in apo E-deficient mice (24). Identifying other biologically significant pathways should permit the rational design of antioxidant therapies designed to inhibit LDL oxidation. Such compounds may block a wide range of oxidative events in the artery wall, with important implications for the prevention of atherosclerotic vascular disease.

1. Witztum, J.L., and Steinberg, D. 1991. Role of oxidized low density lipoprotein in atherogenesis. J. Clin. Invest. 88:1785-1792.

2. Navab, M., et al. 1996. The Yin and Yang of oxidation in the development of the fatty streak. A review based on the 1994 George Lyman Duff Memorial Lecture. Arterioscler. Thromb. Vasc. Biol. 16:831-842.

3. Heinecke, J.W. 1998. Oxidants and antioxidants in the pathogenesis of atherosclerosis: implications for the oxidized low density lipoprotein hypothesis. Atherosclerosis. 141:1-15.

4. Stephens, N.G., et al. 1996. Randomised controlled trial of vitamin $\mathrm{E}$ in patients with coronary disease: Cambridge Heart Antioxidant Study (CHAOS). Lancet. 347:781-786.

5. Heinecke, J.W., Rosen, H., and Chait, A. 1984. Iron and copper promote modification of low density lipoprotein by human arterial smooth muscle cells in culture. J. Clin. Invest. 74:1890-1894.

6. Morel, D.W., DiCorleto, P.E., and Chisolm, G.M. 1984. Endothelial and smooth muscle cells alter low density lipoprotein in vitro by free radical oxidation. Arteriosclerosis. 4:357-364.

7. Steinbrecher, U.P., Parthasarathy, S., Leake, D.S., Witztum, J.L., and Steinberg, D. 1984. Modification of low density lipoprotein by endothelial cells involves lipid peroxidation and degradation of low density lipoprotein phospholipids. Proc. Natl. Acad. Sci. USA. 81:3883-3887.

8. Steinbrecher, U.P., Lougheed, M., Kwan, W.C., and Dirks, M. 1989. Recognition of oxidized low density lipoprotein by the scavenger receptor of macrophages results from derivatization of apolipoprotein B by products of fatty acid peroxidation. J. Biol. Chem. 264:15216-15223.

9. Haberland, M.E., Cheng, L., and Fong, D. 1988. Malondialdehyde-altered protein occurs in atheroma of Watanabe heritable hyperlipidemic rabbits. Science. 241:215-217.

10. Rosenfeld, M.E., Palinski, W., Yla-Herttuala, S., Butler, S., and Witztum, J.L. 1990. Distribution of oxidation specific lipid-protein adducts and apolipoprotein-B in atherosclerotic lesions of varying severity from WHHL rabbits. Arteriosclerosis. 10:336-346.

11. Kita, T., et al. 1987. Probucol prevents the progression of atherosclerosis in Watanabe heritable hyperlipidemic rabbit, an animal model for famil- ial hypercholesterolemia. Proc. Natl. Acad. Sci. USA. 84:5928-5932.

12. Carew, T.E., Schwenke, D.C., and Steinberg, D. 1987. Antiatherogenic effect of probucol unrelated to its hypercholesterolemic effect: evidence that antioxidants in vivo can selectively inhibit low density lipoprotein degradation in macrophage-rich fatty streaks and slow the progression of atherosclerosis in the Watanabe heritable hyperlipidemic rabbit. Proc. Natl. Acad. Sci. USA. 84:7725-7729.

13. Zhang, S.H., et al. 1997. Paradoxical enhancement of atherosclerosis by probucol treatment in apolipoprotein E-deficient mice. J. Clin. Invest. 99:2858-2866.

14. Parthasarathy, S., Young, S.G., Witztum, J.L., Pittman, R.C., and Steinberg, D. 1986. Probucol inhibits oxidative modification of low density lipoprotein. J. Clin. Invest. 77:641-644.

15. Diaz, M.N., Frei, B., Vita, J.A., and Keaney, J.F. 1997. Antioxidants and atherosclerotic heart disease. N. Engl. J. Med. 337:408-416.

16. Fruebis, J., Steinberg, D., Dresel, H.A., and Carew, T.E. 1994. A comparison of the antiatherogenic effects of probucol and of a structural analogue of probucol in low density lipoprotein receptordeficient rabbits. J. Clin. Invest. 94:392-398.

17. Witting, P., et al. 1999. Dissociation of atherogenesis from aortic accumulation of lipid hydroperoxides in Watanabe heritable hyperlipidemic rabbits. J. Clin. Invest. 104:213-220.

18. Kleinveld, H.A., Demacker, P.N.M., and Stalenhoef, A.F.H. 1994. Comparative study on the effect of low-dose vitamin $\mathrm{E}$ and probucol on the susceptibility of LDL to oxidation and the progression of atherosclerosis in Watanabe heritable hyperlipidemic rabbits. Arterioscler. Thromb. 14:1386-1391.

19. Pratico, D., Tangirala, R.K., Rader, D.J., Rokach, J., and FitzGerald, G.A. 1998. Vitamin E sup presses isoprostane generation in vivo and reduces atherosclerosis in ApoE-deficient mice. Nat. Med. 4:1189-1192.

20. Chisolm, G.M., et al. 1994. 7 beta-hydroperoxycholest-5-en-3 beta-ol, a component of human atherosclerotic lesions, is the primary cytotoxin of oxidized human low density lipoprotein. Proc. Natl. Acad. Sci. USA. 91:11452-11456.

21. Watson, A.D., et al. 1997. Structural identification by mass spectrometry of oxidized phospholipids in minimally oxidized low density lipoprotein that induce monocyte/endothelial interactions and evidence for their presence in vivo. J. Biol. Chem. 272:13597-13607.

22. Heinecke, J.W. 1997. Mechanisms of oxidative damage of low density lipoprotein in human atherosclerosis. Curr. Opin. Lipidol. 8:268-274.

23. Leeuwenburgh, C., et al. Mass spectrometric quantification of markers for protein oxidation by tyrosyl radical, copper, and hydroxyl radical in low density lipoprotein isolated from human atherosclerotic plaques. J. Biol. Chem. 272:3520-3526.

24. Cyrus, T., et al. 1999. Disruption of the 12/15 lipoxygenase gene diminishes atherosclerosis in apo E-deficient mice. J. Clin. Invest. 103:1597-1604. 